\title{
Codificação de Fonte e Canal para Transmissão de Imagens em Canal AWGN
}

\author{
Ricardo Barroso Leite e Yuzo Iano
}

\begin{abstract}
Resumo - Para a transmissão de grande quantidade de dados através de canais ruidosos, como é o caso das transmissões via satélite, cuidados devem ser tomados para que a informação seja recebida de forma correta e com o menor custo em relação à largura de banda ocupada. Para atingir esses objetivos, este trabalho apresenta uma comparação de desempenho de diferentes combinações de técnicas de codificação de fonte e técnicas de codificação de cnal, baseadas em transformada wavelet, codificação SPIHT(Set Partitioning In Hierarchical Trees) e códigos Turbo Convolucional. São determinados os melhores casos para a transmissão em canal AWGN (Additive White Gaussian Noise), em termos de PSNR (Peak Signal to Noise Ratio), SSIM (Structural SIMilarity Index).
\end{abstract}

Palavras Chave - Codificação de Fonte, Codificação de Canal, Compressão de imagens, Transmissão via Satélite, SPIHT, Códigos Turbo, SSIM.

Abstract - For the transmission of large amounts of data over noisy channels, such as satellite transmissions, care must be taken so that the information is received as it should and with the lowest cost in terms of bandwidth. To achieve these goals, this paper presents a performance comparison of different combinations of source coding techniques and channel coding techniques, based on wavelet transform, SPIHT coding (Set Partitioning In Hierarchical Trees) and Turbo Convolutional Coding Best cases for transmission in AWGN (Additive White Gaussian Noise) channel, in terms of PSNR (Peak Signal to Noise Ratio), SSIM (Structural SIMilarity Index) are presented.

Keywords- Source Coding, Channel Coding, Image Compression, Satellite Transmission, SPIHT, Turbo Code, SSIM.

\section{INTRODUÇÃO}

As imagens obtidas via satélites artificiais são muito importantes para diferentes áreas de pesquisa, como sensoriamento remoto, astronomia, aplicações militares e meteorológicas. Para garantir que essas imagens sejam recebidas com qualidade considerável e de forma rápida, se tornam necessários métodos de codificação digital que eliminem redundâncias (Codificação de Fonte) e garantam imunidade a ruídos (Codificação de Canal).

O ruído mais importante a ser considerado é o de origem térmica, tanto nas antenas receptoras, quanto nos sistemas de envio do satélite e na atmosfera. Para modelar esses efeitos, costuma se utilizar o ruído aditivo com distribuição gaussiana (AWGN). Com o método de codificação adequado pode-se obter qualidade nos dados recebidos a uma dada taxa, segundo a teoria proposta por Shannon [2].

Esta pesquisa foi financiada pelo Conselho Nacional de Desenvolvimento Científico e Tecnológico (CNPq 102278/2007-5). Os autores estão associados ao Departamento de Comunicações da Faculdade de Engenharia Elétrica e de Computação da Universidade Estadual de Campinas - UNICAMP, 13083-970, São Paulo, Brasil. Participam também do projeto ITASAT - Programa de Satélites Universitários, em um convênio ITA-UNICAMP (e-mails:rleite@fee.unicamp.br,yuzo@decom.fee.unicamp.br).

\section{CODIFICAÇÃO DE FONTE}

Os métodos de codificação se distinguem em dois grupos: a Codificação de Fonte, que tem como objetivo diminuir a quantidade de bits do sinal, eliminando redundâncias na informação, de forma a facilitar a transmissão e o armazenamento (seqüências menores podem ser transmitidas a taxas mais rápidas); e a Codificação de Canal, que por outro lado aumenta a quantidade de bits do sinal, adicionando bits de controle ou paridade, para que seja possível no receptor serem detectados e/ou corrigidos erros introduzidos por interferências e ruídos.

\section{A. Transformada Wavelet Discreta (TWD)}

Uma forma muito usada de se comprimir imagens e preservar as qualidades visuais é através da Transformação Wavelet. Esta análise consiste em aplicar filtros a imagem, de forma a separar as baixas freqüências (alta escala ou coeficientes de aproximação) das altas freqüências (baixa escala ou coeficientes de detalhe), e ao mesmo tempo separá-las também no domínio espacial. Após a filtragem, os sinais obtidos são sub-amostrados (reduzindo pela metade o número de amostras) de forma a conservar o número de amostras do sinal original (Fig. 1). Após esta etapa, os coeficientes de aproximação podem ser novamente filtrados a fim de conseguir melhores resultados. Após a separação em subbandas, os coeficientes wavelet são codificados através do algoritmo SPIHT (Set Partitioning In Hierarchical Trees, [3]). Esse algoritmo permite .o ajuste da taxa de bits de forma precisa, através do truncamento dos coeficientes (quantização bitplane), privilegiando as subbandas mais significativas (aproximação).

Para esse trabalho é usada a função wavelet CDF 9/7, em 3 níveis de filtragem, mesma usada no padrão JPEG 2000 pra a compressão lossy de imagens estáticas [4].

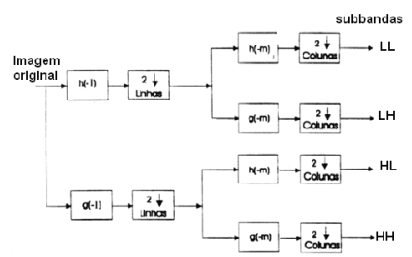

(a)

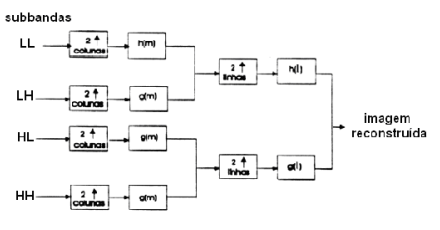

(b)
Fig. 1 - Aplicação da Transformada Wavelet em uma imagem (a) decomposição (b) reconstrução

\section{CODIFICAÇÃO DE CANAL}

\section{A. Turbo Convolucional}

O Código Turbo em Paralelo [5] consiste em colocar dois codificadores convolucionais em paralelo, sendo um deles com a entrada embaralhada por um entrelaçador (Fig. 2). A saída contém bits vindos dos 3 ramos, multiplexados de forma a se obter a taxa de codificação necessária.

A vantagem do esquema Turbo é que, usando dois processos de codificação se consegue maior segurança nos bits de saída. O Entrelaçador ajuda a corrigir erros muito próximos (em rajada ou em burst). Na recepção o algoritmo de Viterbi é implementado de maneira recursiva, de forma a aproveitar as 2 codificações e, após um número de iterações é feita uma decisão. 


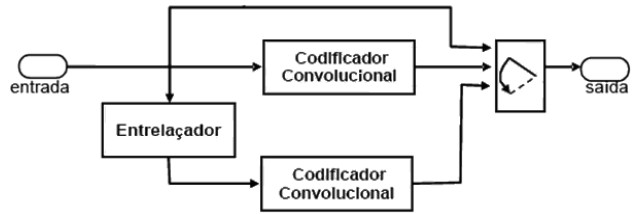

Fig. 2 - Codificador Turbo Convolucional em Paralelo

\section{RESULTADOS EXPERIMENTAIS}

Os modelos de Codificação de Fonte e Canal, juntamente com as operações envolvidas na modulação e condicionamento dos sinais foram implementadas em ambiente de simulação computacional MATLAB Simulink.

A seguir, são apresentadas algumas curvas comparativas entre um dos esquemas de codificação simulados e a imagem transmitida sem codificação. Foi usada a imagem Lena (Fig. 4(a), [10]) para fazer os testes, por se tratar de uma imagem muito utilizada na área de processamento de imagens, o que facilita a comparação. Foi avaliads a relação sinal ruído de pico (PSNR) e também a medida de similaridade estrutural (SSIM - Structural SIMilarity Index, [9]), que se aproxima mais da qualidade visual subjetiva.

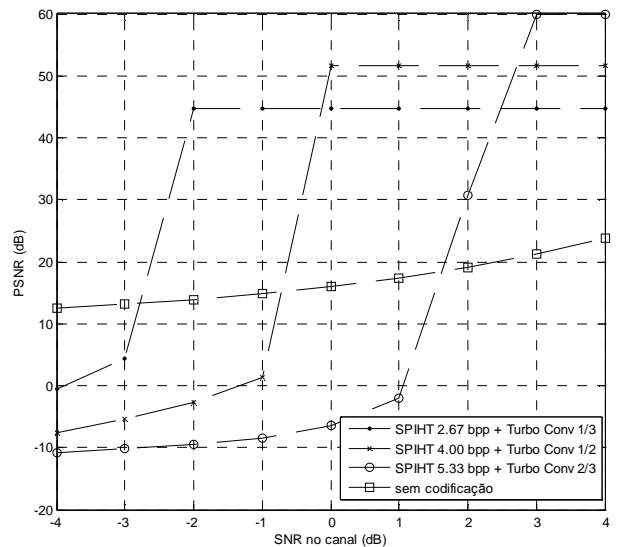

(a)

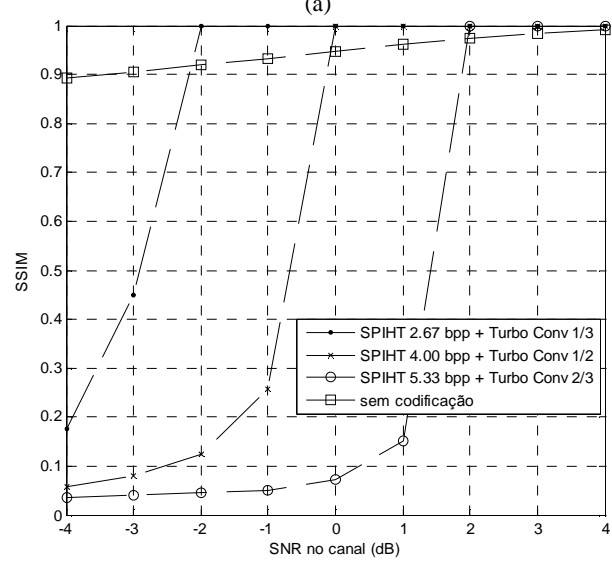

(b)

Fig. 2 - Desempenho da codificação (a) PSNR (b) SSIM

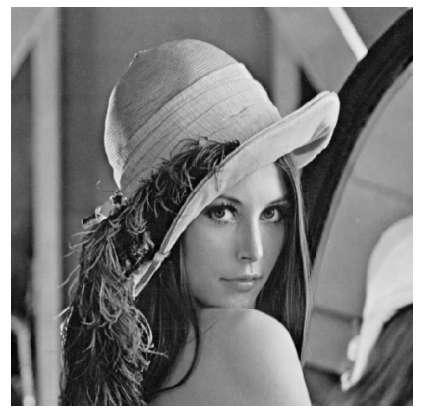

(a)

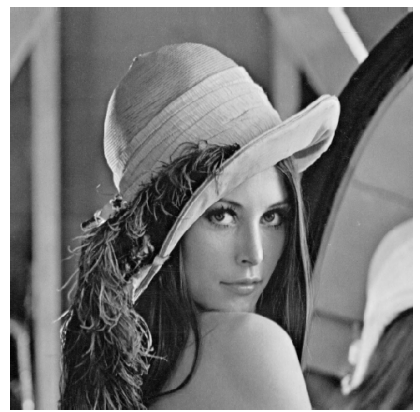

(b)

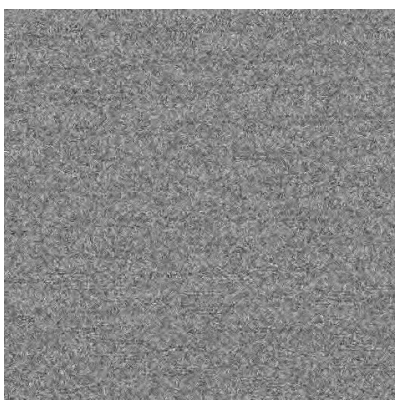

(c)

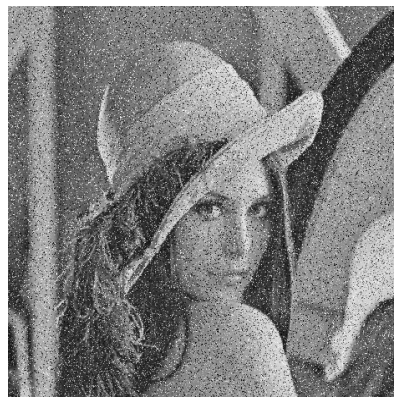

(d)
Fig. 4 - Imagens obtidas com SNR = 0 dB no canal (a) SPIHT 2.67 bpp + Turbo Conv. 1/3 (b) SPIHT 4.00 bpp + Turbo Conv. 1/2 (c) SPIHT 5.67 bpp + Turbo Conv. 2/3 (d) sem codificação

\section{CONCLUSÕES}

Pela análise das curvas e também das imagens decodificadas, observa-se que a codificação digital pode ajudar na recuperação sem erros da imagem na transmissão por um canal ruidoso. Embora a quantidade de bits transmitida seja a mesma, a codificação permite a preservação das componentes mais importantes visualmente e a correção de erros aditivos introduzidos pelo canal. Conclui-se que a distorção devido a redução de bits na codificação de fonte não tem tanta relevância quanto os erros introduzidos no canal. Portanto a melhor solução seria a que utilizasse mais bits para a paridade e maior compressão dos dados da fonte.

\section{AGRADECIMENTOS}

O projeto contou com o apoio dos pesquisadores da Unicamp envolvidos com o projeto ITASAT (Ana Lúcia Mendes Cruz Silvestre da Silva, Fernando Silvestre da Silva, Roger Fredy Larico Chavez, Natasha Bortolozo Menéndez, Alcides da Costa Lino Neto, André Filgueiras de Araújo e Maurício Passador Valério).

Sou muito grato pelo apoio prestado pelo Conselho Nacional de Desenvolvimento Científico e Tecnológico (CNPq) que viabilizou este projeto por meio de bolsa de Iniciação Científica.

Agradeço também todo o apoio prestado pelos órgãos FAPESP (Fundação de Amparo à Pesquisa do Estado de São Paulo), FAEP (Fundação de Apoio ao Ensino e Pesquisa da UNICAMP) e CAPES (Coordenação de Aperfeiçoamento de Pessoal de Nível Superior) pelos anos de apoio às pesquisas desenvolvidas no Laboratório de Comunicações Visuais (LCV) da Unicamp.

\section{REFERÊNCIAS}

[1] Silva, Ana Lúcia Mendes Cruz Silvestre da, Procedimentos para método híbrido de compressão de imagens digitais utilizando transformadas Wavelet e codificação fractal, Universidade Estadual de Campinas, Faculdade de Engenharia Elétrica e de Computação, 2005;

[2] C. E. Shannon, "A mathematical theory of communication," Bell System Technical Journal, vol. 27, pp. 379-423 and 623-656, July and October, 1948;

[3] Amir Said, William A. Pearlman, "A new fast and efficient image coding based on set partitioning in hierarchical trees," IEEE Trans. Circuits, Syst. Video Technol., vol. 6, no. 3, pp. 243-250, Jun. 1996;

[4] JPEG 2000, http://www.jpeg.org/jpeg2000/;

[5] C. Berrou, A. Glavieux, P. Thitimajshima, "Near Shannon Limit Error-Correcting Coding and Decoding Turbo Codes," Proc. Int. Conf. Communications, p. 10641070, Maio 1993;

[6] A. Viterbi and J.Omura, "Principals of Digital Communication and Coding," McGraw-Hill Book Company, New York, USA, 1979;

[7] G. D. Forney, "The Viterbi algorithm," Proceedings of the IEEE 61(3):268-278, Mar 1973;

[8] Wavelet Toolbox, MATLAB - The Language of Technical Computing, The Matworks, Inc., 2004;

[9] Z. Wang, A. C. Bovik, H. R. Sheikh and E. P. Simoncelli, "Image quality assessment: from error visibility to structural similarity," IEEE Transactions on Image Processing, vol. 13, pp. 600-612, Apr., 2004;

[10] Waterloo BragZone test images. Disponível em: ftp://links.uwaterloo.ca/pub/BragZone; 